\title{
Design of Smart Environmental Protection Supervision System in the COVID-19 Post-epidemic Era
}

\author{
PengfeiGuo,Jianwei Lu \\ Huzhou University, Huzhou, Zhejiang, China
}

\begin{abstract}
Objectives: This research proposes a smart environmental protection monitoring system based on the Internet of Things technology, which is used to solve the monitoring data collection of the source of waste discharge of enterprises and organizations, as well as the monitoring of waste transportation and real-time monitoring at the end of waste treatment.Methods: The system covers the whole-process big data of environmental protection from waste classification and reuse, collection and transportation, online trading, production control to product traceability and government supervision. Results: Realizes unmanned supervision.Conclusions: Fills the gap in waste classification supervision in China.
\end{abstract}

Keywords: epidemic era, smart environmental protection, environmental protection supervision, Internet of Things, garbage classification

\section{Introduction}

With the spread of the COVID-19 pandemic, China's environmental protection management model is shifting from the goal of pollution control to the goal of environmental quality improvement. There is still a clear gap in the existing environmental monitoring models and capabilities in adapting to this transformation. In recent years, environmental protection informatization has received attention from the government and environmental protection departments. The State Council clearly stated in the "Decision on Implementing the Scientific Outlook on Development and Strengthening Environmental Protection": "We must improve the environmental monitoring network, build the 'Golden Ring Project', and realize "Environmental protection, speed up the construction of environmental and nuclear safety information systems, and implement information resource sharing mechanisms".

\section{Research Status}

In recent years, there have been a few research results on smart environmental protection supervision at home and abroad, and they are mainly concentrated in three aspects:

First, research from the status quo of the application of smart environmental protection systems in smart parks: Zhang Chunguang proposed based on the "digital environmental protection" platform and IoT technology in the article "Analysis of the application of smart environmental protection systems in smart parks" (2016) that the in-depth development in the field of environmental protection and the establishment of a nationwide Internet of Things system in the field of environmental protection is the first step to realize the transformation from "digital environmental protection" to "smart environmental protection"[1].

Second, research from the aspect of digital environmental protection: Yang Xuejun believes that the development trend of digital environmental protection is intelligent in the article "Construction and Research of an Integrated Platform for Digital Environmental Protection Based on Intelligence-Taking Shenzhen as an Example" (2015). Under the background, the construction ideas and research of digital environmental protection integrated platform are very important in environmental protection management innovation. His paper proposes the basic framework of digital environmental protection and establishes a detailed analysis and system platform to integrate the Internet of Things with mobile law enforcement, resource integration, and model application.Through the actual application

ISSN: 0010-8189

(c) CONVERTER 2021

www.converter-magazine.info 
in environmental supervision and early warning, it has promoted the innovation of environmental management models[2].

Third, research from the application of data mining technology in smart environmental protection systems: Gao Guikang's "Application Research of Data Mining Technology in Desulfurization Monitoring Smart Environmental Protection System" (2016) believes that through the use of data mining technology, and association rule algorithm to analyze data mining, which can effectively find out the connection between the data to improve the comprehensive decision-making ability and provide a reliable basis for smart environmental protection[3].

The existing smart environmental protection supervision (information) system design is designed only for the collection of environment-related data, such as the collection, detection and early warning of waste water and waste gas discharge data. There is no monitoring data collection for the source of waste classification and discharge of the company, and no monitoring on the way of garbage transportation and real-time supervision of the end of the garbage disposal. There is a regulatory gap.

\section{System Function Design}

\subsection{Public service platform system}

The system is divided into PC and mobile apps, which can provide garbage source companies and individuals with a recyclable garbage trading platform. The system can find corresponding recycling companies or individuals through various matching conditions and realize transactions as soon as possible. It can create a new industrial system for closed-loop waste disposal.

\subsection{Garbage collection vehicle management and control system}

The front end of the system can comprehensively monitor information such as the real-time location, frequency of operation, operation trajectory, number of coverage, operation status, operation specifications, violations and other information of the transportation operation vehicle by installing environmental protection transportation vehicle sensor monitoring unit equipment, conduct dispatch management of vehicles in emergency situations, control the operation data of all clearing vehicles in real time, perform statistical analysis of vehicle operation data through real-time calculation on the cloud platform, and provide data support for functional department supervision and dispatch decision-making.

\subsection{Waste classification supervision platform system}

The system takes comprehensive asset management as the core, budget management as the main line, production and operation as the basis, performance appraisal as the standard, standardizes and optimizes business processes, and analyzes the supply, demand, production, and management chain. It formulates a standard garbage classification mechanism data model, and uses advanced and practical integration, platform, and humanized design concepts for design and development to ensure a high degree of data sharing and uniformity, flexible and efficient operation, comprehensive cost control, and maximum recycling to achieve economic benefits, realizing a new industrial system of "closed-loop" waste disposal under the background of circular economy.

\subsection{Environment real-time monitoring platform}

The system provides powerful big data visualization analysis functions. By automatically extracting, combining and analyzing and mining various historical data in the environmental protection system, it displays intuitively and visually to provide data support for managers' decision-making. It supports multi-dimensional, dynamic and flexible drilling, and friendly user interaction. 


\subsection{Integrated real-time monitoring system}

The integrated real-time monitoring system can realize the real-time three-dimensional visual display of the location, trajectory, event, etc. of urban garbage removal vehicles and personnel. It provides reliable and intuitive decision support for real-time scheduling and optimized configuration of environmental resources. Using the map as a carrier, it displays the emission parameters of various waste incineration plants in real time. It conducts real-time monitoring and alarm for abnormal emission enterprises. At the same time, it carries out correlation analysis and comprehensive display in combination with environmental parameters such as air quality.

\section{System Overall Design}

The system adopts a design scheme that combines software and hardware:

(1) Install garbage disposal source installation layout in each major enterprise in the jurisdiction based on NB-IoT (Narrow Band Internet of Things) or 5G network communication technology sensor monitoring unit for comprehensive monitoring. The sensor detection unit includes a high-definition network digital camera, a temperature and humidity sensor, a sound sensor, and a dust meter (including three parameters of PM2.5, PM10 and TSP dust). In this way, it realizes the monitoring of enterprise waste classification and all-round monitoring from the source of waste disposal.

(2)Install sensor monitoring units based on 5G mobile network communication technology for garbage transportation vehicles, including high-definition network digital cameras and GPS satellite positioning modules, to achieve full route monitoring of garbage transportation vehicles and real-time vehicle status monitoring, and urge the standardization of garbage transportation vehicles operation.

(3) Comprehensively monitor the sensor monitoring unit based on NB-IoT (Narrow Band Internet of Things) or 5G network communication technology in the installation layout of garbage terminal processing enterprises. The sensor detection unit includes high-definition network digital cameras, temperature Humidity sensor, sound sensor, dust meter (including three parameters of PM2.5, PM10 and TSP dust). In this way, the standardized operation of waste terminal treatment enterprises can be supervised and urged.

The sensor monitoring unit stores the collected real-time information on the cloud server platform through the information conversion module, router, and coordinator, and transmits it to the application layer after processing through cloud computing, GIS cloud services, data mining, and visualization technologies on the cloud platform. The application layer is divided into an intranet platform used by government regulatory agencies and an extranet platform open to the public. The functional modules of the intranet platform include: a comprehensive real-time monitoring platform, a real-time environmental monitoring platform, a pollution emergency command platform, and a garbage classification monitoring platform. The functional modules of the external network platform include: a public service platform and a public government affairs platform.

The public service platform is divided into PC terminal and mobile terminal APP, which can provide garbage source companies and individuals with a recyclable garbage trading platform, and create a new closed-loop garbage disposal industry system.

The real-time environmental monitoring system adopts a big data visualization analysis system, which provides powerful big data visualization analysis functions. By automatically extracting, combining and analyzing and mining various historical data in the environmental protection system, it is displayed in an intuitive visualization for management Decision-making to provide data support. It supports multi-dimensional, dynamic and flexible drilling, and friendly user interaction.

ISSN: 0010-8189

(C) CONVERTER 2021 
The integrated real-time monitoring platform realizes the real-time three-dimensional visual display of the location, trajectory, and events of garbage collection vehicles and personnel through the integrated real-time monitoring system. It can provide reliable and intuitive decision support for real-time scheduling and optimized configuration of environmental resources. It uses the map as the carrier to display the emission parameters of each waste incineration plant in real time, and conduct real-time monitoring and alarm for abnormal emission enterprises. At the same time, it carries out correlation analysis and comprehensive display in combination with environmental parameters such as air quality. It supports mainstream large-screen display devices and various VR helmet devices. The overall design of the system is shown in Figure 1: 


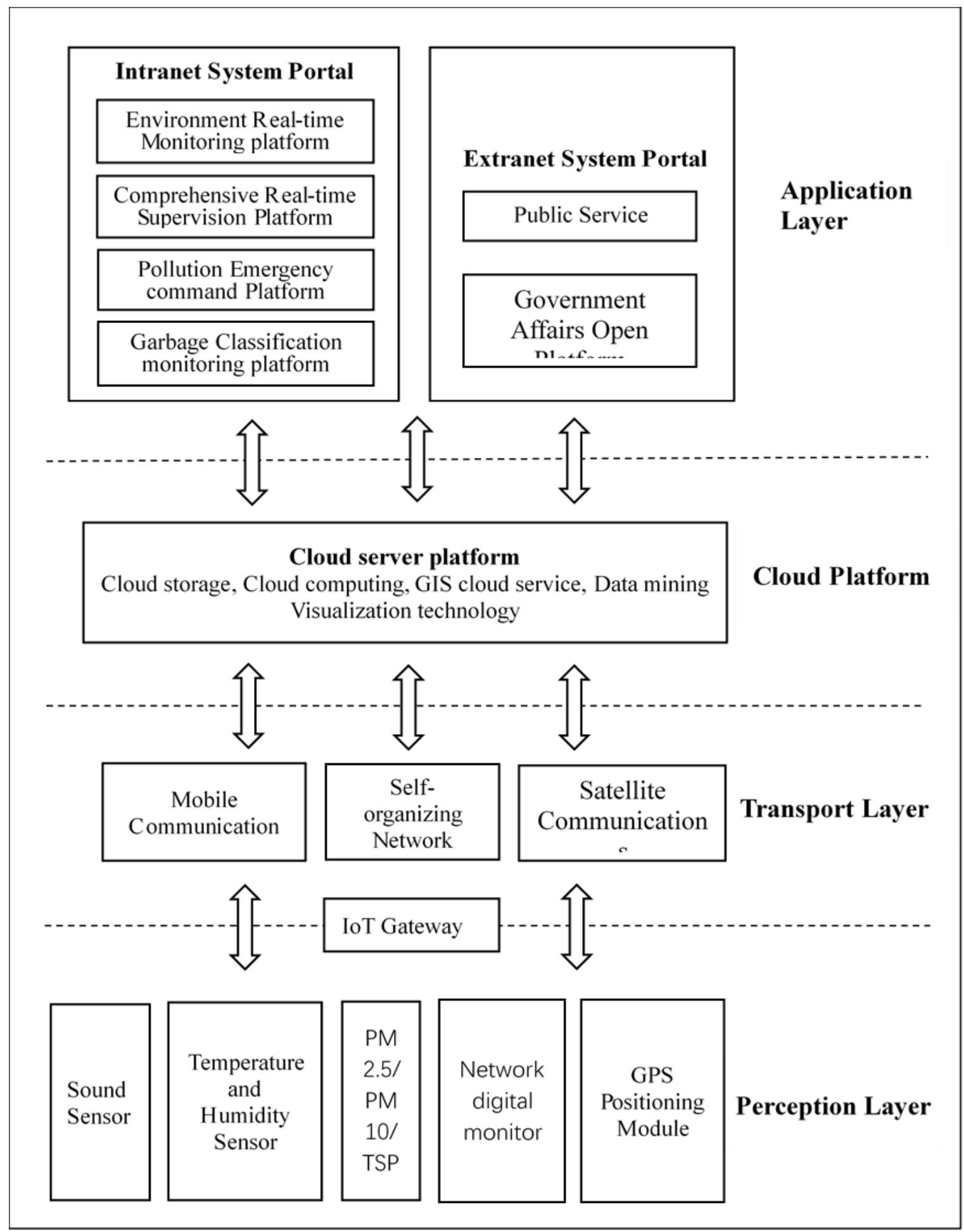

Fig 1: Schematic diagram of the overall structure of the system

\section{System Detailed Design}

5.1 Hardware design of environmental information collection

The environmental collection hardware is composed of temperature and humidity sensors, sound sensors, dust meters (including PM2.5, PM10 and TSP dust parameters) and a single-chip microcomputer. The DQ end of the

ISSN: 0010-8189

(C) CONVERTER 2021

www.converter-magazine.info 
DS18B20 digital temperature and humidity sensor is connected to the P1.2 of the single-chip microcomputer. The single-chip microcomputer communicates with the temperature sensor through serial high and low level signals to read temperature information. The OUT terminal of the sound sensor is connected to the P1.0 of the single-chip microcomputer. The system can adjust the sensitivity of the sensor through the variable resistor on the sound sensor. When the environmental sound decibel is below the sensitivity, the OUT terminal of the sound sensor outputs a high level. When the environment is too noisy, the sound decibel will be above the sensitivity, then the OUT terminal of the sound sensor will output a low level[4]. The OUT terminal of the dust sensor is connected to the P1.1 of the single-chip microcomputer. The system can also adjust the variable resistor on the sensor to adjust the sensitivity of the dust sensor. When the dust concentration in the environment exceeds the sensitivity of the sensor, the level of the dust sensor OUT will change from high to low. The single-chip microcomputer scans the temperature, sound, and dust information cyclically. When the serial port is interrupted when receiving the network request, it will send the current scanned temperature, sound, and dust information through the serial port. The hardware connection of environmental information collection is shown in Figure 2:

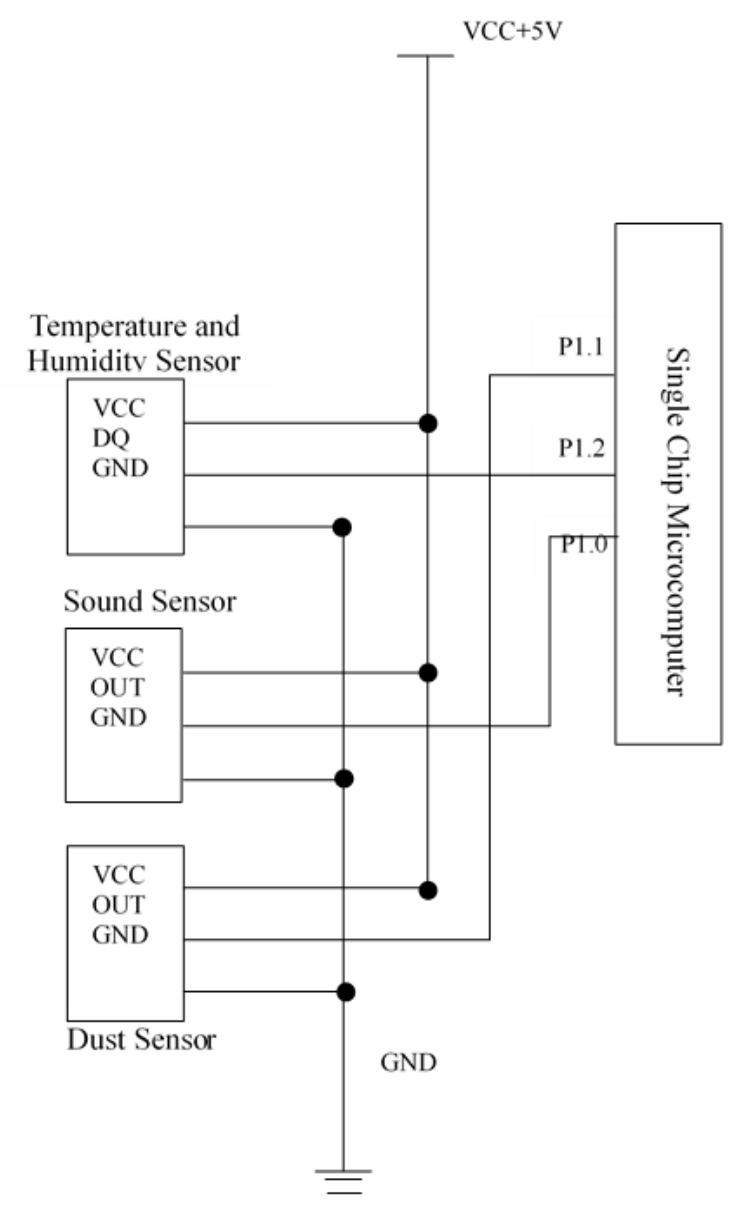

Fig 2: Schematic diagram of the hardware structure of environmental information collection

5.2 Network digital camera and positioning hardware structure design

The network high-definition camera and positioning hardware structure consists of network digital cameras, GPS positioning modules, switches, and routers. The network digital camera has the video and image acquisition kinetic energy of the traditional camera, and also has a digital image compression controller and a network-oriented operating system. It can compress and encrypt the video image data and transmit it to the terminal through the Ethernet or wireless network.

ISSN: 0010-8189

(C) CONVERTER 2021 
The access of network digital camera and positioning hardware structure can be used to monitor the whole process of distinguishing garbage classification, real-time monitoring of the environment of garbage removal vehicles, and video monitoring of the entire operation of garbage incineration plants-- the garbage terminal processing companies. At the same time, the obtained monitoring point location information is directly used for coordinate display on the map, realizing the real-time 3D visualization display of the location, trajectory, event, etc. of urban garbage removal vehicles and personnel, which provides reliable real-time scheduling and optimized configuration of environmental protection resources, Intuitive decision support. It uses the map as the carrier to display the emission parameters of each waste incineration plant in real time, and conduct real-time monitoring and alarm for abnormal emission enterprises. At the same time, it carries out correlation analysis and comprehensive display in combination with environmental parameters such as air quality. The connection of network HD camera and positioning hardware is shown in Figure 3:

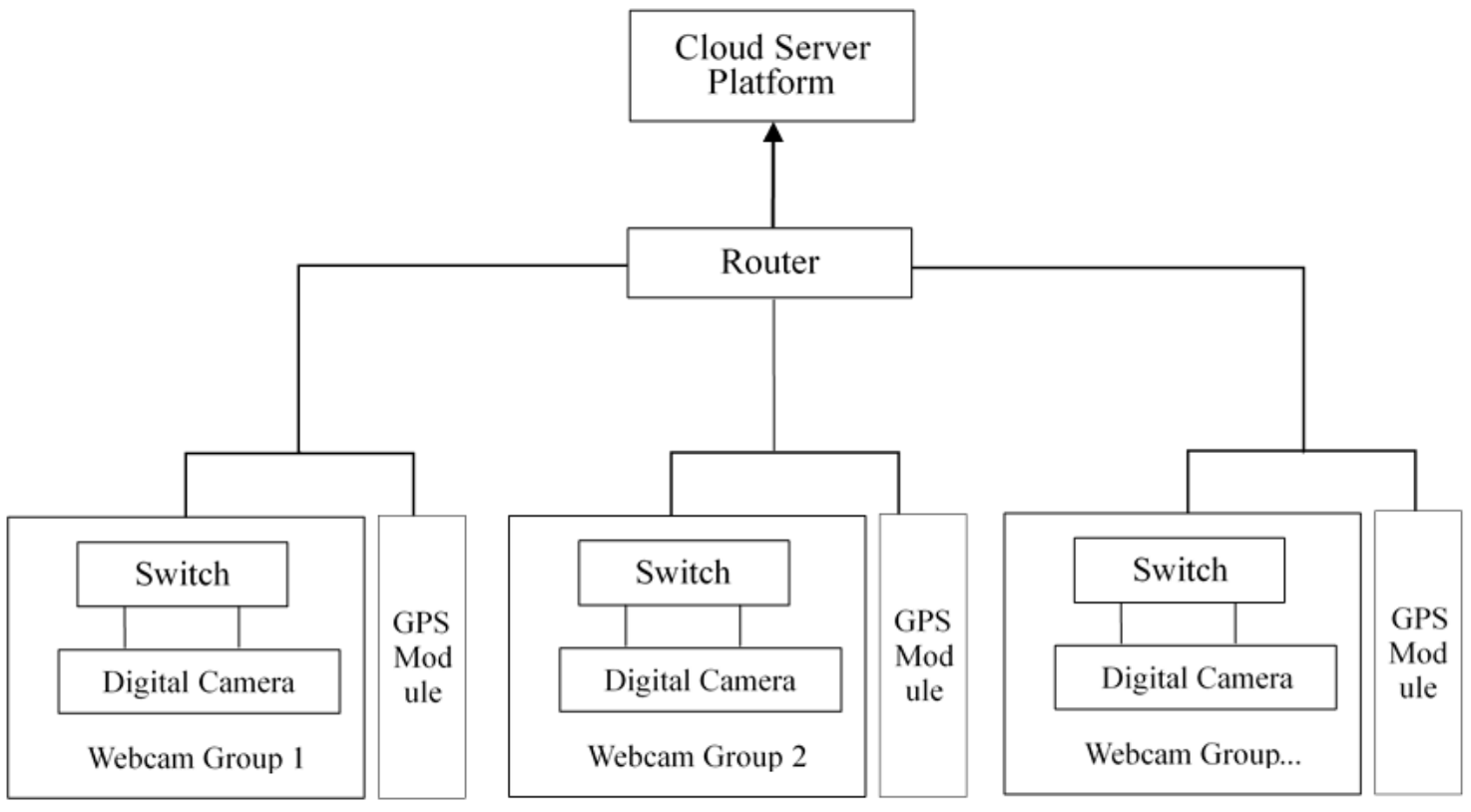

Fig 3: Schematic diagram of network HD camera and positioning hardware structure

\section{Specific Implementation Process}

\subsection{Sensor unit implementation process}

The first sensor detection unit installed at the source of garbage disposal of the enterprise is used to monitor the garbage classification of the enterprise; the second sensor detection unit installed on the garbage transportation vehicle is used to monitor the route and status of the garbage transportation vehicle Monitoring; the third sensor detection unit set at the garbage terminal treatment site is used to monitor the standardized operation of the garbage terminal treatment site.

The first sensor detection unit includes a camera (high-definition network digital camera), a temperature and humidity sensor, a sound sensor, and a dust meter (including three parameters of PM2.5, PM10, and TSP dust). They monitor the image, temperature, humidity, sound, and dust of the company's waste treatment from the source, which can realize the monitoring of corporate waste classification and comprehensive monitoring from the source of the waste treatment; the first sensor detection unit adopts the narrowband Internet of Things Or the 5G communication network and transmits the collected data to the cloud server platform.

ISSN: 0010-8189 
The second sensor detection unit includes a camera (high-definition network digital camera) and a GPS satellite positioning module, which is used to monitor the position of the garbage transport vehicle and the state in the vehicle in real time, and supervise the standardized operation of the garbage transport vehicle; the second sensor detection unit of the $5 \mathrm{G}$ mobile communication network is used to transmit the collected data to the cloud server platform.

The front end of the system can comprehensively monitor information such as the real-time location, operating frequency, operating trajectory, coverage times, operating status, operating specifications, violations and other information of transport operating vehicles through the installation of environmentally-friendly transportation vehicle sensor detection unit equipment, and conduct dispatching management of vehicles under emergency situations. It can control the operating data of all clearing and transportation vehicles in real time, and perform statistical analysis of vehicle operation data through real-time calculation on the cloud platform, and provide data support for functional department supervision and scheduling decision-making.

The third sensor detection unit includes a camera (high-definition network digital camera), a temperature and humidity sensor, a sound sensor, and a dust meter (including PM2.5, PM10, and TSP dust), which can monitor the image, temperature, humidity, sound and dust of the garbage terminal treatment, and supervise the standardized operations of end-of-garbage disposal companies; the third sensor detection unit uses narrowband Internet of Things or $5 \mathrm{G}$ communication network to transmit the collected data to the cloud server platform.

\subsection{Application layer implementation process}

The cloud server platform performs different processing on the data uploaded by each sensor detection unit and transmits it to the application layer; the application layer includes the intranet system portal and the extranet system portal. The intranet system portal displays the data of all sensor detection units for operation User supervision; the external network system portal provides preset and publicly available data for users to view.

(1) The intranet system portal includes a comprehensive real-time monitoring platform, a real-time environmental monitoring platform, a pollution emergency command platform, and a garbage classification monitoring platform The comprehensive real-time supervision platform uses the map as the carrier to visually display the data detected by the three sensing detection units on the map according to the location; realize the real-time three-dimensional visual display of the location, trajectory, event, etc. of garbage removal vehicles and personnel. It provides reliable and intuitive decision support for real-time scheduling and optimized configuration of environmental resources. It uses the map as a carrier to display the emission parameters of various waste incineration plants in real time, monitor and alarm abnormal emission companies in real time, and perform correlation analysis and comprehensive display in combination with environmental parameters such as air quality. The platform supports mainstream large-screen display devices and various VR helmet devices;

The real-time environmental monitoring platform is used to store and display the historical data of the three sensing detection units; the real-time environmental monitoring platform adopts a big data visualization analysis system, provides powerful big data visualization analysis functions, and automatically performs various historical data' s extraction, integration, analysis and mining in the environmental protection system, providing data support for managers' decision-making with intuitive visualization. It supports multi-dimensional, dynamic and flexible drilling, and friendly user interaction;

The pollution emergency command platform conducts environmental protection public opinion detection and early warning based on Internet media big data and collected real-time data; it is convenient for supervisory departments and enterprises to detect sudden public opinions in time and track the progress of public opinions. At the same time, it can dispatch and command emergency incident handling in an all-round way;

ISSN: 0010-8189

C CONVERTER 2021

www.converter-magazine.info 
The waste classification supervision platform combines the data collected by the first sensing detection unit to supervise the waste classification operation process of the waste classification source enterprises. Using integrated, platform-based, and humanized design concepts, and based on ecological theory, a standard garbage classification mechanism data model can be established (the existing published garbage classification standards can be used). The monitoring personnel can refer to the given model or standard on the platform, and monitor the operation process of the enterprise's waste classification source based on the data collected by the first sensing detection unit.

(2) The extranet system portal includes a public service platform and a public government affairs platform

The government affairs disclosure platform is used to display preset public government affairs information to users;

The public service platform includes PC terminal and mobile terminal APP. It is a recyclable garbage trading platform, and the information on recyclable garbage is disclosed on it. Users input demand conditions through the platform, and the platform obtains corresponding recyclable garbage for users to recycle according to the matching conditions. The platform can provide a recyclable garbage trading platform for garbage source companies and individuals, and create a new closed-loop garbage disposal industry system.

\section{Conclusions}

Compared with the existing technology, this system has the following innovations: First, it collects monitoring data on the source of waste discharge from the enterprise organization, monitors the waste transportation, and conducts real-time monitoring at the end of the waste treatment, which complements the existing technology. The second is that the system displays real-time monitoring data using maps as a carrier to facilitate supervision by monitoring personnel; the third is that the system provides a recyclable garbage trading platform to achieve "closed-loop" garbage in the context of a circular economy Deal with the new industrial system. The third is that the system provides a trading platform for recyclable garbage and realizes a new industrial system of "closed-loop" garbage disposal in the context of a circular economy.

\section{References}

[1] X.J. Yang, Y.H. Zhou, “The construction and research of an integrated platform for digital environmental protection based on intelligence-taking Shenzhen as an example," Environment, no. S1, pp. 10-12, 2015.

[2] G.K. Gao, "Application research of data mining technology in desulfurization monitoring intelligent environmental protection system," Hangzhou Dianzi University, 2016.

[3] C.G. Zhang, D.X. Zhang, "Analysis of the application of smart environmental protection system in smart parks,” Smart Buildings and Smart Cities, no. 04, pp. 62-65,2016.

[4] P.F. Guo, C.J. Gong, G. Wang, "Design of smart reservation parking system based on ZigBee technology," Journal of Shandong Normal University (Natural Science Edition), vol. 33, no. 03, pp. 302-308, 2018. 